\title{
PERCEPÇÃO DE COORDENADORES DE SAÚDE BUCAL SOBRE A REDE DE ATENÇÃO À SAÚDE BUCAL
}

\section{Perception of oral health coordinators on the health care network}

\section{Brunna Verna Castro Gondinho}

Faculdade de Odontologia de Piracicaba - FOP/UNICAMP - Piracicaba (SP) Brasil

\section{Luciane Miranda Guerra}

Faculdade de Odontologia de Piracicaba - FOP/UNICAMP - Piracicaba (SP) Brasil

\section{Jaqueline Vilela Bulgareli}

Faculdade de Odontologia de Piracicaba - FOP/UNICAMP - Piracicaba (SP) Brasil

\section{Lívia Fernandes Probst}

Faculdade de Odontologia de Piracicaba - FOP/UNICAMP - Piracicaba (SP) Brasil

\section{Karine Laura Cortellazzi}

Faculdade de Odontologia de Piracicaba - FOP/UNICAMP - Piracicaba (SP) Brasil

\section{Rosana de Fátima Possobon}

Faculdade de Odontologia de Piracicaba - FOP/UNICAMP - Piracicaba (SP) Brasil

\section{Antonio Carlos Pereira}

Faculdade de Odontologia de Piracicaba - FOP/UNICAMP - Piracicaba (SP) Brasil

\section{Vinícius Alexandre da Silva Oliveira}

Universidade Estadual do Piauí - UESPI - Teresina (PI) - Brasil

\section{RESUMO}

Objetivo: Aprofundar a temática "Redes de atenção à saúde bucal", suas dificuldades de implantação e sugestões para sua operacionalização, segundo as percepções de coordenadores de saúde bucal. Métodos: Estudo de natureza qualiquantitativa, realizado com os quatro gestores das cidades, sedes das macrorregiões de saúde do Piauí, que responderam a questões referentes ao tema, sua realidade, dificuldades e sugestões. As respostas foram analisadas através do discurso do sujeito coletivo. Resultados: Os gestores pontuaram possíveis caminhos para a implementação da rede temática da saúde bucal e dificuldades a serem superadas: falta de adequação do setor de saúde às necessidades territoriais, precária estruturação dos níveis de atenção à saúde, insuficiência de recursos humanos e necessidade de maior conhecimento em relação às redes de atenção à saúde. Conclusão: Salienta-se que segundo os gestores inquiridos é possível a operacionalização do eixo temático saúde bucal em um contexto de implantação das Redes de Atenção à Saúde, ao mesmo tempo em que é preciso que sejam considerados os obstáculos assinalados para que seja possível construir os meios de superação dos mesmos.

Descritores: Gestão em Saúde; Assistência à Saúde; Saúde Bucal.

\begin{abstract}
Objective: The aim of this study was to deepen the theme "Oral health care networks", their implementation difficulties and suggestions for its operationalization, according to the perceptions of oral health coordinators. Methods: were the qualitative and quantitative study carried out with the four managers of the cities, headquarters of health macro-regions of Piauí, who answered questions related to the theme, their reality, difficulties and suggestions. Responses were analysed using the collective subject discourse. Results: The results show that the managers pointed out possible paths for the implementation of the thematic network of oral health and difficulties to be overcome: inadequacy of the health sector to territorial needs, precarious structuring of levels of health care, insufficient human resources and need of knowledge regarding health care networks. Conclusion: according to the managers interviewed, it is possible to operationalize the thematic axis of oral health in a context of implementation of the health care networks, while it is necessary to consider the obstacles identified so that it is possible to build the means of overcoming them.
\end{abstract}

Descriptors: Health Management; Delivery of Health Care; Oral Health. 


\section{INTRODUÇÃO}

Sistemas de Atenção à Saúde podem ser compreendidos como o conjunto de atividades que objetivam promover, restaurar e manter equitativamente a saúde de uma população ${ }^{(1)}$. Para serem organizados de maneira resolutiva torna-se indispensável conhecer e analisar as necessidades de saúde da população, a partir dos seus aspectos demográficos e epidemiológicos ${ }^{(2,3)}$.

Constitui-se em um problema fundamental a ser superado pelo Sistema Único de Saúde (SUS) a sua estrutura e organização frente à acelerada transição demográfica brasileira, concomitantemente à complexa transição epidemiológica. Para essa finalidade justifica-se a construção de um modelo pautado na promoção da saúde e na definição de novos espaços de cuidado e de planejamento participativo. Na prática, idenficica-se uma série de desafios estruturais e ideológicos que vão pela contramão da história, que sempre apresentou organizações institucionais piramidais, verticais e médico-centradas ${ }^{(4)}$.

A solução passa, portanto, pela descentralização dos serviços do SUS, tendo por alicerces os princípios de regionalização e de hierarquização. Para esse alcance, propõe-se a implementação de uma modelagem que possibilite uma organização articulada e integrada das ações e serviços de saúde, ofertados entre os níveis de atenção do sistema, ou seja, a estruturação das Redes de Atenção à Saúde (RAS) ${ }^{(5)}$.

As RAS podem ser definidas como organizações poliárquicas, com pontos vinculados entre si e com objetivos comuns, atuando por meio de ações cooperativas e interdependentes, permitindo ofertar uma atenção contínua e integral à determinada população, sob a coordenação da atenção básica à saúde. Desta forma, o sistema em rede organiza-se sob a horizontalidade de atenção à saúde e tem por centro de comunicação a atenção primária em saúde(6).

No âmbito da saúde bucal, ações para a reorganização da atenção ocorrem desde 2004, por meio da Política Nacional de Saúde Bucal( ${ }^{(7)}$. Desde então, houve uma ampliação da atenção à saúde bucal nos níveis secundário e terciário ${ }^{(8)}$. Todavia esse tema ainda é pouco debatido na literatura. Dessa forma, percebe-se que a temática da saúde bucal nas RAS é uma discussão que deve ganhar espaço no debate organizativo, partindo de aspectos da gestão até questões referentes ao processo de trabalho dos profissionais que exercem a odontologia, em razão de sua importância para a integração e articulação dos pontos de atenção à saúde. Dessa forma se deseja contribuir para a efetiva implantação de um modelo de atenção resolutivo( ${ }^{(9)}$.

Frente ao contexto, a inserção da temática Saúde Bucal nas discussões sobre RAS é de suma importância, para a efetiva organização de um modelo de atenção que tenha por objetivo a integralidade do cuidado. Debate-se uma sincronizada articulação entre os seus pontos e níveis. Neste momento a Saúde Bucal precisa assumir um papel protagonista em saúde, deixando, portanto, de ser uma mera participante incluída em outras temáticas trabalhadas, para ser a própria temática discutida e estruturada em rede.

Assim, o objetivo deste estudo é aprofundar quanto à temática das redes de atenção à saúde bucal, suas dificuldades de implantação e sugestões para sua operacionalização, segundo as percepções de coordenadores de saúde bucal.

\section{MÉTODOS}

Trata-se de ser realizado um censo de natureza qualiquantitativa, com os 4 coordenadores municipais de saúde bucal, dos respectivos municípios, sedes das macrorregiões de saúde do Estado do Piauí.

A pesquisa foi realizada nesse Estado, onde o processo de regionalização se equaciona atendendo às suas reais condições financeiras. Pauta-se na ideia que haja uma clara definição de prioridades de investimentos que possibilitem o ordenamento efetivo dos territórios bem como, o funcionamento qualificado e resolutivo do Sistema Estadual de Saúde. Não se deixam de contemplar os aspectos técnicos e legais, preconizados nos instrumentos em vigor que norteiam a organização e o funcionamento do SUS. Além dessas questões, também se rearranja tendo por base norteadora a insuficiência de dispositivos públicos de saúde com condições infraestruturais disponíveis no interior do Estado. São inúmeros os vazios assistenciais identificados em toda a extensão territorial do Estado e existe uma rarefação na distribuição populacional ${ }^{(10)}$.

Atualmente, o Piauí organiza-se em 11 Regiões de Saúde - Territórios de desenvolvimento (Figura 1), que são referência para a média complexidade; e em 4 Macrorregiões (Figura 2) que são referência para a alta complexidade ${ }^{(10)}$. 


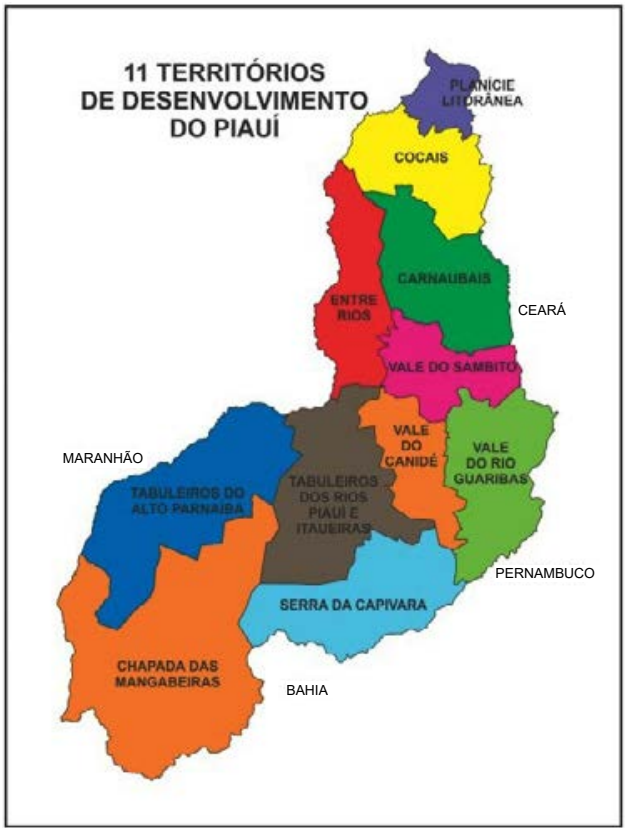

Figura 1 - Territórios de Desenvolvimento - Regiões de Saúde do Piauí.

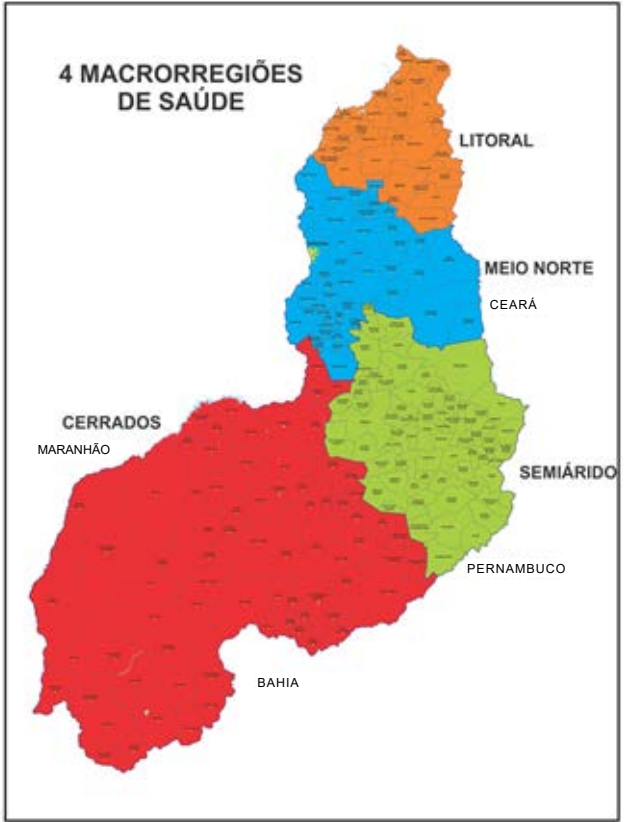

Figura 2 - Macrorregiões de Saúde do Piauí.

Os coordenadores receberam via e-mail o Termo de Consentimento Livre e Esclarecido (TCLE), juntamente com um questionário contendo as questões abertas da pesquisa.

Para a análise das respostas enviadas por e-mail, utilizou-se a técnica de processamento de dados do discurso do sujeito coletivo (DSC). Destaca-se que o DSC é uma proposta qualiquantitativa para pesquisa de opinião ou representação social, que engloba depoimentos sintetizados, analisados e redigidos na primeira pessoa do singular, bem como, expressa o pensamento coletivo por meio do discurso dos sujeitos. Esta técnica parte da hipótese de que, em sociedade, as pessoas compartilham ideias, opiniões, crenças ou representações, apresentando dupla pertinência: qualitativa e quantitativa. A qualitativa, porque cada opinião coletiva é representada sob a forma de um discurso e recupera os distintos conteúdos e argumentos que formam uma dada opinião na escala social, e quantitativa, pois o discurso tem uma representação numérica indicando quantos depoimentos semelhantes cada DSC obteve $\mathrm{e}^{(11,12)}$. 
Além dos discursos, uma pesquisa desta natureza apresenta também o grau de compartilhamento (GC), que expressa, quantitativamente, a proporção de pessoas que compartilham ideia semelhante e as ideias centrais (IC) identificadas. As IC são expressões linguísticas que descrevem, de maneira sintética e precisa, o sentido das expressões chave. Entretanto, não se trata da interpretação, mas sim de uma descrição do conteúdo de cada uma dessas expressões chave. Tais resultados são obtidos através de uma série de operações de organização de dados sob a forma de texto, auxiliadas pelo software Qualiquantisoft(13).

Desta forma, a técnica de DSC tem como fundamentos as representações sociais que se referem a um conjunto de crenças, proposições, falas e condutas que se institucionalizam e se tornam rotineiros no cotidiano das comunicações pessoais ${ }^{(14)}$.

O presente trabalho foi aprovado pelo Comitê de Ética em Pesquisa da Universidade Estadual do Piauí - UESPI, sob o Parecer de número 1.115.358.

\section{RESULTADOS E DISCUSSÃO}

Nas Tabelas I, II e III são apresentados os dados quantitativos, a partir da síntese das ideias centrais e suas respectivas frequências de manifestações nas respostas. Em seguida, estão dispostos os resultados expressos pelos respectivos Discursos do Sujeito Coletivo (DSC) produzidos.

A seguir são apresentados os discursos do sujeito coletivo referente às ideias centrais da Tabela I.

Tabela I - Síntese das ideias centrais e respectiva frequência de manifestações para a questão "Discorra sobre o tema 'Rede de Atenção à Saúde Bucal' relacionando-o com a sua realidade".

\begin{tabular}{llc}
\hline Ideias Centrais & $\mathbf{n}$ & \% \\
\hline A - Possibilidade de implementação & 4 & $100 \%$ \\
B - Dificuldades existentes & 3 & $75 \%$ \\
\hline
\end{tabular}

Obs.: A soma da frequência de ideias centrais pode extrapolar o número de 4 respostas e a porcentagem de $100 \%$, na medida em que um indivíduo pode apresentar mais de uma ideia central.

Discurso do sujeito coletivo da ideia central A - Possibilidade de implementação:

"Acredito que a Rede de Atenção à Saúde Bucal possa ser implementada (...) seria algo bastante importante para a nossa realidade (...) poderá acontecer se tivermos um conjunto organizado de serviços, com articulação inter setorial, Redes de Atenção guiadas pela missão de garantir o cuidado integral e longitudinal em saúde (...) vejo que a saúde bucal precisa ir além de uma organização de fluxos e protocolos diluídos nas demais redes temáticas já em implementação, e isso poderá acontecer por meio da Rede de Atenção à Saúde Bucal como uma prerrogativa nacional. Assim defendo que a criação desta rede temática específica da saúde bucal junto ao Ministério da Saúde é importante para o fortalecimento do Programa Brasil Sorridente e do Sistema Único de Saúde como um todo (...) o trabalhar em rede é o caminho para a resolutividade."

O discurso acima traz uma ideia positiva a respeito da saúde bucal como temática das RAS, ao mesmo tempo em que ressalta a necessidade de uma organização articulada e inter setorial dos serviços, na garantia pelo direito à saúde em uma perspectiva de integralidade do cuidado, fato que corrobora os estudos de Pucca Jr ${ }^{(15)}$. Segundo o mesmo pesquisador, um dos grandes desafios da odontologia é se identificar como uma área dessa integralidade do SUS, organizando-se a partir daí em uma Rede de Atenção à Saúde, que supere as especificidades odontobiológicas.

Somado a essa orientação, tem-se o atual cenário da Política de Saúde Bucal no Brasil, que é de avanços e que aponta para os indícios favoráveis quanto ao efeito de redução das iniquidades em saúde, mesmo diante das dificuldades iniciais de implantação do atendimento público odontológico com amplitude universal. Essa abordagem também permite que os serviços públicos odontológicos abram espaço para ações de promoção da saúde, por meio da expansão da atenção primária, educação em saúde, ações preventivas e levantamentos epidemiológicos ${ }^{(16)}$.

Nesta perspectiva, compreende-se que os serviços públicos deem sinais de rompimento com o paradigma "odontocentrado". Observa-se esse rompimento, quando se expõem destaques e experiências positivas da inserção da odontologia na Estratégia Saúde da Família. Outro sinal passa pela situação em que se ampliam as formas de acesso às linhas de cuidado e níveis tecnológicos em saúde bucal a partir da possibilidade de regulação do cuidado. Um outro indicador é quando se permite uma formação profissional voltada para um modelo de atenção de base 
territorial. Consequentemente, há de se supor que o modelo de atenção em saúde bucal provavelmente opere com melhor capacidade de resposta às expectativas e às necessidades individuais e coletivas, quando organizado em rede ${ }^{(17)}$.

Esse resultado, portanto, reforça a indispensabilidade da saúde bucal assumir um protagonismo para fortalecimento de sua política, deixando de ser apenas um ponto a ser trabalhado por outras temáticas em seus fluxos e protocolos.

Discurso do sujeito coletivo da ideia central B - Dificuldades existentes

“Em nosso município e regional de saúde o trabalho em rede ainda não está bem definido (...) é preciso, antes de qualquer coisa, haver uma sensibilização de profissionais e gestores para o reconhecimento das necessidades territoriais locais (...) temos em nossa regional déficit nos níveis de atenção em saúde, são dificuldades estruturais, baixo número de profissionais e de qualificação para um trabalho em rede e no SUS (...) a meu ver, só será possível estabelecer a implementação das Redes de Atenção, isso também incluindo a Rede de Atenção à Saúde Bucal como temática, quando houver, por parte dos níveis de gestão, um estudo direcionado a cada regional, identificando carências e potencialidades, para assim trabalhar a questão da comunicação entre os níveis de atenção, qualificar e estruturar serviços, estabelecendo linhas de cuidado, incentivar e tornar capaz o pessoal que trabalha no Sistema Único de Saúde."

Os gestores entrevistados neste estudo reconhecem e elencam as dificuldades em relação às Redes de Atenção à Saúde e à Rede de Atenção à Saúde Bucal, enquanto temática. Ao passo que enfatizam como possíveis certos caminhos para a superação desses obstáculos, fazem o reconhecimento das necessidades territoriais, identificando, para tal, suas dificuldades e possibilidades. Desta forma se estabelecerem linhas de cuidado e se trabalham os canais de comunicação entre os pontos da rede em seus níveis de atenção. Segundo Milton Santos ${ }^{(18)}$, o território é um objeto vivo, dinâmico e compreende não só as características físicas de uma determinada área, como também integra as inter-relações e as marcas produzidas pelos seres humanos. Assim, os pesquisados, ao reconhecerem a importância deste desbravamento territorial, defendem nada mais que a territorialização como um instrumento organizativo relevante dos processos de trabalho e das práticas em saúde, questão postulada por Monken e Barcellos $^{(19)}$ em seu estudo.

Em relação às linhas de cuidado, essas precisam ser entendidas para além de um mero fluxo que serve de instrumento gestor e favorece o estabelecimento de protocolos clínicos. Necessitam ser reconfiguradas para que, além de orientarem o percurso dos usuários dentro do sistema e dentro dos próprios serviços, levem em consideração as relações oriundas desse percurso ${ }^{(20)}$, ou seja, levem à formulação da dimensão micropolítica e relacional do trabalho em saúde(21).

Outro ponto sinalizado pelos pesquisados é a qualificação para o trabalho no SUS. Em relação a estes aspectos da formação para o SUS, Ceccim e Feuerwerker ${ }^{(22)}$, destacaram a necessidade da criação, por parte dos Ministérios da Saúde e Educação, de políticas públicas para a educação e formação dos profissionais, sustentadas nos princípios e diretrizes do SUS. Dessa forma será de permitir e direcionar a execução de ações com capacidade de trocas entre o ensino em saúde, a gestão setorial, as práticas de atenção e o controle social em saúde (quadrilátero da formação em saúde).

Assim colocado, a formação dos profissionais de saúde pode passar a ser visualizada como um projeto educativo que extrapolaria o domínio técnico-científico da profissão, adentrando nos aspectos que estruturam as relações e práticas dos componentes de relevância social. Essa orientação certamente contribuiria com a elevação da qualidade do serviço prestado à população. Acresce dizer que a saúde da população não seja considerada somente em relação aos aspectos epidemiológicos do processo saúde-doença, como também em ligação com os aspectos da gestão setorial e, em especial, com a estruturação de um cuidado em saúde oriundo de uma construção coletiva de atores sociais empoderados.

Tabela II - Síntese das ideias centrais e respectiva frequência de manifestações para a questão "Em sua opinião, existem dificuldades para a Saúde Bucal atuar em Redes de Atenção à Saúde?".

\begin{tabular}{llc}
\hline Ideias Centrais & $\mathbf{n}$ & \% \\
\hline A - Necessidade de equipamentos e procedimentos & 2 & $50 \%$ \\
B - Existem dificuldades para além das tecnológicas & 3 & $75 \%$ \\
\hline
\end{tabular}

Obs.: A soma da frequência de ideias centrais pode extrapolar o número de 4 respostas e a porcentagem de $100 \%$, na medida em que um indivíduo pode apresentar mais de uma ideia central. 
A seguir são apresentados os discursos do sujeito coletivo referente às ideias centrais da Tabela II.

Discurso do sujeito coletivo da ideia central A - Necessidade de equipamentos e procedimentos:

"Sim. Precisamos de estrutura física, equipamentos para os consultórios odontológicos e ampliar o número de procedimentos em âmbito hospitalar."

Discurso do sujeito coletivo da ideia central B - Existem dificuldades para além das tecnológicas:

"Sim. As dificuldades são desde uma nova ideologia na formação do dentista a problemas de incentivos e prioridades pelos gestores locais para as ações e serviços de saúde bucal, principalmente, diante de um subfinanciamento existente. Outro ponto é a dependência tecnológica que o profissional da odontologia possui (...) identifico uma necessidade de um adequado funcionamento da Atenção Primária à Saúde, Atenção Secundária à Saúde e Terciária (...) me chama a atenção a pouca implementação de Unidades de Pronto Atendimento com cirurgiões-dentistas (...) mas também não adianta organizar o serviço, se os profissionais não souberem referenciar.

Historicamente, a odontologia forma profissionais com pensamento tecnicista e influenciados pelo modelo da odontologia de mercado. Esses profissionais associam a alta tecnologia e a vasta gama de equipamentos com procedimentos à saúde. Narvai e Frazão ${ }^{(23)}$ mencionam a necessidade de uma formação em odontologia menos tecno-centrada e mais voltada para a atenção em saúde, ou seja, orientada para que os cirurgiões-dentistas adentrem novos espaços e utilizem condutas para além das restritamente ligadas à existência de equipamentos e ao quantitativo ambulatorial. Tal ideia vai de encontro ao discurso $A$ supra referido, enquanto serve como base para o discurso $B$ também acima descrito.

Igualmente, o segundo discurso atesta o que Narvai(24) defendeu no que diz respeito à integralidade de atenção em saúde, uma vez que para o autor é um erro reduzir a Política Nacional de Saúde Bucal a uma rede de atenção básica, ou a uma rede de Centros de Especialidades Odontológicas (CEOs). É preciso ir além dessas medidas e articular sistematicamente os níveis de atenção à saúde e seus respectivos dispositivos.

Outro ponto destacado é a relevância de um conhecimento, por parte dos atores do processo, a respeito do que venha a ser regulação em saúde. Como defendido por Souza et al ${ }^{(25)}$, analisar a saúde bucal em uma perspectiva de rede aponta para uma necessidade de que os serviços tornem realidade os protocolos de regulação, gerando como resultados a melhoria do acesso e a qualidade da atenção prestada.

Assim, a Rede de Atenção em Saúde Bucal também deve ser coordenada pela atenção primária em saúde, centro de comunicação entre os vários pontos que compõem as redes de cuidado em saúde ${ }^{(26)}$; sendo representada pela Equipe de Saúde Bucal inserida na Estratégia Saúde da Família. Contudo, percebe-se a necessidade de uma reflexão acerca da estruturação da saúde bucal à luz da Estratégia de Saúde da Família e sua dinâmica cogestora regional, já que o perfil do profissional da odontologia, historicamente, é voltado para a prática clínica e para procedimentos pontuais ${ }^{(27)}$.

A seguir é apresentado o discurso do sujeito coletivo referente à ideia central da Tabela III.

Tabela III - Síntese das ideias centrais e respectiva frequência de manifestações para a questão "Quais suas sugestões para auxiliar que a Saúde Bucal atue em uma perspectiva de Rede de Atenção à Saúde?".

\begin{tabular}{llc}
\hline Ideias Centrais & $\mathbf{n}$ & $\mathbf{\%}$ \\
\hline A - É preciso entender as redes de atenção à saúde e incentivar a Saúde Bucal & 4 & $100 \%$
\end{tabular}

Obs.: A soma da frequência de ideias centrais pode extrapolar o número de 4 respostas e a porcentagem de $100 \%$, na medida em que um indivíduo pode apresentar mais de uma ideia central

Discurso do sujeito coletivo da ideia central A - É preciso entender as redes de atenção à saúde e incentivar a Saúde Bucal.

"Entender as Redes de Atenção à Saúde é ponto de partida para o incentivo de implementação da temática Saúde Bucal (...) Precisamos, por meio do conhecimento e da vivência, captar a essência e a dinâmica de operacionalização do que são essas redes, para desta maneira incitar o projeto do eixo da Saúde Bucal, tão relevante para o estímulo à integralidade do cuidado e da atenção em saúde".

Esse discurso corrobora as posições defendidas por Silva(28), em que se refere a RAS. Em seu estudo esse pesquisador defendeu as RAS como uma fornecedora de condições estruturais mais adequadas para a obtenção 
de uma efetiva integralidade da atenção. Nessa abordagem reforça o mencionado por Godoi ${ }^{9}$ ao garantir que sejam necessárias mudanças Rede de Atenção à Saúde Bucal:

"Os avanços para consolidação da rede de atenção à saúde bucal dependem da melhora na definição e organização do elemento população, por meio do levantamento das necessidades desta, seguido de planejamento de base territorial, a fim de que se adotem critérios epidemiológicos e sociais para priorização da atenção à saúde bucal. Para tanto, destaca-se, em relação à estrutura operacional, a ampliação e redefinição da rede mediante maior e melhor dimensionamento dos recursos físicos e humanos, em todos os níveis de atenção; é, ainda, fundamental a qualificação dos sistemas logísticos e de governança, promovendo a interligação entre os pontos de atenção e dos sistemas, de um lado, e toda a gestão da rede de atenção à saúde bucal, de outro. Ressalta-se, também, a efetivação de um modelo de atenção à saúde coerente com a situação de saúde da população, fundamentando-o em princípios de vigilância à saúde" (Godoi, 2014 pag: 330).

Porém, são recentes as publicações do Ministério da Saúde (Resoluções $n^{0} 2^{4}$ e $n^{0} 3^{5}$, de 16 de agosto de 2016. Dispõem, respectivamente, sobre os indicadores para o processo nacional de pactuação inter federativa relativo ao ano de 2016 e se constitui um subgrupo de trabalho tripartite. Esse subgrupo cria-se no âmbito do grupo de trabalho de gestão da comissão inter gestores tripartite, com a finalidade de elaborar proposta de revisão do Decreto $n^{0} 7.508$, de 28 de junho de 2011). Pensa-se que essa equipe poderá sinalizar um caminho de possível ameaça aos avanços oriundos, tanto da Política Nacional de Saúde Bucal, como do processo de organização do SUS, em suas Redes - RAS.

\section{CONCLUSÃO}

Observou-se que os coordenadores atribuíram importância ao eixo da Saúde Bucal enquanto temática das RAS. Colocaram também a possibilidade de sua viabilização, ao mesmo tempo em que foram assinalados obstáculos e os seus possíveis meios de superação.

Este estudo contribui para o debate a respeito da organização das ações de saúde bucal, uma vez que foram alcançados pontos considerados fundamentais para uma melhor organização do cuidado de saúde nas realidades estudadas.

Tais constatações são de interesse da sociedade, dos trabalhadores do SUS e da gestão em saúde. Com esses resultados pode-se discutir o problema com os atores envolvidos, a fim de aprimorar a atenção prestada ao público usuário do sistema.

\section{AGRADECIMENTOS E CONFLITOS DE INTERESSE}

Agradecimentos à Secretaria de Saúde do Estado do Piauí pelo apoio institucional e logístico a esta pesquisa. Os autores informam que não há relações que possam implicar potencial conflito de interesse.

\section{REFERÊNCIAS}

1. World Health Organization. The world health report 2000: health systems, improving performance. Geneva: WHO, 2000.

2. Frenk, J. Bridging the divide: comprehensive reform to improve health in Mexico. Nairobi, Comission on Social Determinants of Health, 2006.

3. Mendes, EV. As redes de atenção à saúde. Ciênc Saude Coletiva. 2010;15(5):2297-305.

4. Paim, J; Travassos, C; Almeida, C; Bahia, L.; Macinko, J. O sistema de saúde brasileiro: história, avanços e desafios. The Lancet, Saúde no Brasil maio de 2011, p.11-31.

5. Lavras $C$. Descentralização, regionalização e estruturação de redes regionais de atenção à saúde no SUS. In: Ibanez N, Elias PEM, Seixas PHD, organizadores. Política e Gestão Pública em Saúde. São Paulo: Hucitec Editora: Cealag, 2011.

6. Mendes, EV. As redes de atenção à saúde. Brasília: Organização Pan-Americana da Saúde; 2011.

7. Brasil. Ministério da Saúde. Diretrizes da Política Nacional de Saúde BucaL Coordenação Nacional de Saúde Bucal. Brasília: Ministério da Saúde; 2004. 
8. Brasil. Departamento de Atenção Básica, Secretaria de Atenção à Saúde, Ministério da Saúde. Cadernos de atenção básica 17: saúde bucal. Brasília: Ministério da Saúde; 2006. (Série A. Normas e Manuais Técnicos).

9. Godoi, H; Mello, ALSF; Caetano, JC. Rede de atenção à saúde bucal: organização em municípios de grande porte de Santa Catarina, Brasil. Cad. Saúde Pública, Rio de Janeiro, 30(2):318-332, fev, 2014.

10. Secretaria Estadual de Saúde do Piauí. Atualização do Plano Diretor de Regionalização do Estado do Piauí (PDR)- Pi: Diretoria de Unidade de Planejamento - DUP Piauí: 2015.20.p.

11. Lefèvre F, Lefèvre AMC. O sujeito coletivo que fala. Interface-Comunic, Saúde, Educ. 2006; 10(20):517-524 [acesso em 2010 Nov 18]. Disponível em: http://www.scielo.br/pdf/icse/v10n20/17.pdf.

12. Lefèvre F, Lefèvre AMC. Pesquisa de representação social. Um enfoque qualiquantitativo. Brasília: Líber Livro editora; 2012.

13. Lefèvre F, Lefèvre AMC. O discurso do sujeito Coletivo. Caxias do Sul: Editora Educs; 2003.

14. Minayo MCS. O desfio do conhecimento: pesquisa qualitativa em saúde. São Paulo: Hucitec; 2007.

15. Pucca Jr, GA. Saúde bucal: a construção de um bem universal. Revista Espaço Acadêmico; n. 122; Jul: 2011; 109-110.

16. Antunes JLF \& Narvai PC. Políticas de saúde bucal do Brasil. Rev Saúde Pública 2010;44(2):360-5.

17. Mello ALSF et al. Saúde bucal na rede de atenção e processo de regionalização. Ciência \& Saúde Coletiva, 19(1):205-214, 2014.

18. Santos M. O país distorcido: o Brasil, a globalização e a cidadania. São Paulo: Publifolha; 2002.

19. Monken, M; Barcellos, C. Vigilância em saúde e território utilizado: possibilidades teóricas e metodológicas. Cad. Saúde Pública, Rio de Janeiro, 21(3):898-906, mai-jun, 2005.

20. Silva, NEK; Sancho, LG; Figueiredo, WS. Entre fluxos e projetos terapêuticos: revisitando as noções de linhas de cuidado em saúde e itinerários terapêuticos. Ciência \& Saúde Coletiva, 21(3): 843-851, 2016.

21. Malta, DC; Merhy, EE. O percurso da linha do cuidado sob a perspectiva das doenças crônicas não transmissíveis. Interface (Botucatu) 2010; 34(14): 593-606.

22. Ceccim, RB; Feuerwerker, LCM. O Quadrilátero da Formação para a Área da Saúde: Ensino, Gestão, Atenção e Controle Social. PHYSIS: Rev. Saúde Coletiva, Rio de Janeiro, 14(1):41- 65, 2004.

23. Narvai, PC; Frazão, P. Saúde bucal no Brasil: muito além do céu da boca. Rio de Janeiro: Fiocruz, 2008.

24. Narvai, PC. Avanços e desafios da Política Nacional de Saúde Bucal no Brasil. Tempus - Actas de Saúde Colet, 2011; 5 (3), 21-34.

25. Souza, GC; Lopes, MLDS; Roncalli, AG; Medeiros-Júnior, A; Clara-Costa, IC. Referência e contra referência em saúde bucal: regulação do acesso aos centros de especialidades odontológicas. Rev. salud pública. 17 (3): 416-428, 2015.

26. Cecilio, LCO; Andreazza, R; Carapinheiro, G; Araújo, ECA; Oliveira, LA; Andrade, MGG et al. AAtenção Básica à Saúde e a construção das redes temáticas de saúde: qual pode ser o seu papel?. Ciência \& Saúde Coletiva, 17(11):2893-2902, 2012.

27. Martelli, P. J. L. et al. Análise do modelo de atenção à saúde bucal em municípios do estado de Pernambuco. Ciência \& Saúde Coletiva, 13(5):1669-1674, 2008.

28. Silva, SF. Organização de redes regionalizadas e integradas de atenção à saúde: desafios do Sistema Único de Saúde (Brasil). Ciência \& Saúde Coletiva, 16(6): 2753-2762, 2011.

\section{Endereço para correspondência:}

Brunna Verna Castro Gondinho

Av. Limeira, 901

Bairro Areião

CEP: 13.414-903 - Piracicaba - SP - Brasil.

E-mail: bvernagondim@hotmail.com 